


\section{ICASE Fluid Mechanics}

Due to increasing research being conducted at ICASE in the field of fluid mechanics, future ICASE reports in this area of research will be printed with a green cover. Applied and numerical mathematics reports will have the familiar blue cover, while computer science reports will have yellow covers. In all other aspects the reports will remain the same; in particular, they will continue to be submitted to the appropriate journals or conferences for formal publication. 


\title{
BOUNDARY LAYER FLOW OF AIR OVER WATER ON A FLAT PLATE
}

\author{
1st Lt John Nelson ${ }^{1}$ \\ United States Air Force Wright Laboratories \\ Wright-Patterson Air Force Base, $\mathrm{OH}$ 45433-7913 \\ Amy E. Alving ${ }^{2}$ \\ Department of Aerospace Engineering and Mechanics \\ University of Minnesota \\ 110 Union Street S.E. \\ Minneapolis, MN 55455 \\ Daniel D. Joseph \\ Department of Aerospace Engineering and Mechanics \\ and The Minnesota Supercomputer Institute \\ University of Minnesota \\ 110 Union Street S.E. \\ Minneapolis, MN 55455
}

\begin{abstract}
A non-similar boundary layer theory for air blowing over a water layer on a flat plate is formulated and studied as a two-fluid problem in which the position of the interface is unknown. The problem is considered at large Reynolds number (based on $x$ ), away from the leading edge. We derive a simple non-similar analytic solution of the problem for which the interface height is proportional to $x^{1 / 4}$ and the water and air flow satisfy the Blasius boundary layer equations, with a linear profile in the water and a Blasius profile in the air. Numerical studies of the initial value problem suggests that this asymptotic, non-similar air-water boundary layer solution is a global attractor for all initial conditions.
\end{abstract}

\footnotetext{
${ }^{1}$ The work of the first and third authors was supported by the National Aeronautics and Space Administration under NASA Contract No. NAS1-19480 while they were in residence at the Institute for Computer Applications in Science and Engineering (ICASE), NASA Langley Research Center, Hampton, VA 23681. The work was also supported by the National Science Foundation, the DOE, Department of Basic Energy Sciences, the U.S. Army, Mathematics and AHPCRC, and the Minnesota Supercomputer Center. The first author was also supported by the U. S. Air Force, Wright Laboratories, Flight Dynamics Directorate.

${ }^{2}$ This author's work was supported by the National Science Foundation.
} 
$-$ 


\section{Introduction}

The effects of water layers driven over solid surfaces by wind are of interest in the performance of aircraft in rain, for the de-icing of airplane wings and surely in many other applications. Since such problems are intrinsically of a boundary layer type and since the more interesting phenomenon which might arise, like the formation of waves, film rupture and the like are probably best framed in terms of stability, it is necessary to derive the analytic forms that such flows will take on when instability is neglected. This derivation is carried out here.

Previous works related to the present one are by Yih (1990), who modeled the de-icing problem, and by Wang (1992), who considered the development of boundary layers in the shearing flow of one fluid over another. Both works are flawed by assuming rather than finding the shape of the interface. In the case treated by Wang, the interface is flat but the jump of the normal stress is not zero. Yih assumed that flow in the air is a Blasius flow and the flow in the water is a simple shear. This is a correct form for the boundary layer, as we shall show, but he neglects the variation of film thickness with $x$ and so cannot enforce the kinematic condition at the interface or find its shape.

In this work, we formulate a non-similar boundary layer theory retaining all terms which decay faster than $1 / \xi, \xi=x\left(U / 2 \nu_{2} x\right)^{1 / 2}$, where $\nu_{2}$ is the kinematic viscosity of air. All of the interface conditions are enforced in this asymptotic regime. An effect of the small but non-zero vertical velocity component at the interface is to force the interface to grow like $x^{1 / 4}$ when the boundary layer in the air grows like $x^{1 / 2}$. The interface looks thin on the scale of the boundary layer. Asymptotically, at large $x$, the water and air satisfy the Blasius boundary layer equations with a linear profile in the water and the flat plate profile in the air. This non-similar (or coupled self-similar) solution appears to be a global attractor for all initial conditions.

\section{Governing Equations}

A water film of height $y=h(x)$ is flowing on a flat plate driven by shear stresses emanating from an air stream with streaming velocity $U$. We seek the nature of the flow under the circumstances which give rise to Blasius boundary layers in the flow of one fluid over a flat plate. This motivates the introduction of the same scales that are used in the classical case, giving

$$
\begin{aligned}
\xi & =\left(\frac{U x}{2 \nu_{2}}\right)^{1 / 2} \\
\eta & =y\left(\frac{U}{2 \nu_{2} x}\right)^{1 / 2} \\
\eta^{*} & =h(x)\left(\frac{U}{2 \nu_{2} x}\right)^{1 / 2} \\
u & =\frac{u}{U} \\
v & =v\left(\frac{2 x}{U \nu_{2}}\right)^{1 / 2}
\end{aligned}
$$




$$
\begin{aligned}
\tau & =\frac{t U^{2}}{\nu_{2}} \\
p & =\frac{p}{\rho_{2} U^{2}} \\
f & =\frac{\psi}{\left(2 \nu_{2} x U\right)^{1 / 2}}
\end{aligned}
$$

where $u=f^{\prime}$ is the $x$ component of velocity, $v=\eta f^{\prime}-f-\xi \frac{\partial f}{\partial \xi}$ is the $y$ component if velocity, $\tau$ is the time, $p$ is the pressure and $f$ is the stream function. The parameters $\rho_{2}$ and $\nu_{2}$ are for air; subscript 1 is for water.

These scales are introduced into the Navier-Stokes equations which are written below in the new variables without approximation. The continuity equation becomes

$$
\frac{\partial v}{\partial \eta}-\eta \frac{\partial u}{\partial \eta}+\xi \frac{\partial u}{\partial \xi}=0
$$

The momentum equations are

$$
\begin{aligned}
& \frac{\partial u}{\partial \tau}-\frac{\eta}{4 \xi^{2}} u \frac{\partial u}{\partial \eta}+\frac{1}{4 \xi} u \frac{\partial u}{\partial \xi}+\frac{1}{4 \xi^{2}} v \frac{\partial u}{\partial \eta}= \\
& -\frac{1}{\rho}\left(-\frac{\eta}{4 \xi^{2}} \frac{\partial p}{\partial \eta}+\frac{1}{4 \xi} \frac{\partial p}{\partial \xi}\right)+\nu\left(\left(\frac{1}{4 \xi^{2}}+\frac{\eta^{2}}{16 \xi^{4}}\right) \frac{\partial^{2} u}{\partial \eta^{2}}+\frac{1}{16 \xi^{2}} \frac{\partial^{2} u}{\partial \xi^{2}}+\frac{3 \eta}{16 \xi^{4}} \frac{\partial u}{\partial \eta}-\frac{1}{16 \xi^{3}} \frac{\partial u}{\partial \xi}\right) \\
& \quad \frac{1}{\xi^{2}} \frac{\partial v}{\partial \tau}-\frac{\eta}{4 \xi^{4}} u \frac{\partial v}{\partial \eta}+\frac{1}{4 \xi^{3}} u \frac{\partial v}{\partial \xi}-\frac{u v}{4 \xi^{4}}+\frac{1}{4 \xi^{4}} v \frac{\partial v}{\partial \eta}= \\
& -\frac{1}{\rho} \frac{1}{\xi^{2}} \frac{\partial p}{\partial \eta}+\nu\left(\left(\frac{1}{4 \xi^{4}}+\frac{\eta^{2}}{16 \xi^{6}}\right) \frac{\partial^{2} v}{\partial \eta^{2}}+\frac{1}{16 \xi^{4}} \frac{\partial^{2} v}{\partial \xi^{2}}+\frac{5 \eta}{16 \xi^{6}} \frac{\partial v}{\partial \eta}-\frac{3}{16 \xi^{5}} \frac{\partial v}{\partial \xi}+\frac{3 v}{16 \xi^{6}}\right)
\end{aligned}
$$

In the interface equations written below, [.] designates the jump $(.)_{1}-(.)_{2}$. At the interface $\eta=\eta^{*}(y=h(x))$, the velocity is continuous,

$$
\begin{aligned}
& {[u]=0,} \\
& {[v]=0 .}
\end{aligned}
$$

The shear stress is also continuous across $\eta=\eta^{*}$,

$$
\left[\mu\left(\frac{1}{2 \xi}\left(\frac{\eta^{*}}{\xi}+\frac{\partial \eta^{*}}{\partial \xi}\right) \frac{\partial v}{\partial \eta}+\frac{1}{2}\left(1-\frac{1}{2}\left(\frac{\eta^{*}}{\xi}+\frac{\partial \eta^{*}}{\partial \xi}\right)\right)\left(\frac{\partial u}{\partial \eta}-\frac{1}{4 \xi^{2}} v-\frac{\eta^{*}}{4 \xi^{2}} \frac{\partial v}{\partial \eta}+\frac{1}{4 \xi} \frac{\partial v}{\partial \xi}\right)\right)\right]=0 .
$$

The jump in the normal stress is balanced by interfacial tension,

$$
\begin{gathered}
{\left[p-\rho G \eta^{*}-\frac{2 \mu}{\left(1+\left(\frac{\eta^{*}}{2 \xi}+\frac{1}{2} \frac{\partial \eta^{*}}{\partial \xi}\right)^{2}\right.}\left(1-\left(\frac{\eta^{*}}{2 \xi}+\frac{1}{2} \frac{\partial \eta^{*}}{\partial \xi}\right)^{2}\right) \frac{1}{4 \xi^{2}} \frac{\partial v}{\partial \eta}\right.} \\
\left.\left.-\left(\frac{\eta^{*}}{2 \xi}+\frac{1}{2} \frac{\partial \eta^{*}}{\partial \xi}\right)\left(\frac{1}{2 \xi} \frac{\partial u}{\partial \eta}+\frac{1}{8 \xi^{2}} \frac{\partial v}{\partial \xi}-\frac{\eta^{*}}{8 \xi^{3}} \frac{\partial v}{\partial \eta}-\frac{1}{8 \xi^{3}} v\right)\right)\right] \\
+\frac{S}{\left(1+\left(\frac{\eta^{*}}{2 \xi}+\frac{1}{2} \frac{\partial \eta^{*}}{\partial \xi}\right)^{2}\right)^{3 / 2}}\left(\frac{1}{8 \xi} \frac{\partial^{2} \eta^{*}}{\partial \xi^{2}}+\frac{1}{8 \xi^{2}} \frac{\partial \eta^{*}}{\partial \xi}-\frac{1}{8 \xi^{3}} \eta^{*}\right)=0,
\end{gathered}
$$


where $G=\left(g / U^{2}\right)\left(2 \nu_{2} x / U\right)^{1 / 2}, g$ is the acceleration due to gravity, $S=T /\left(\nu_{2} \rho_{2} U\right)$, and $T$ is the coefficient of surface tension. Density and viscosity are normalized by the corresponding properties of the air

$$
\begin{aligned}
& \rho= \begin{cases}\frac{\rho_{1}}{\rho_{2}} & 0 \leq \eta<\eta^{*} \\
1 & \eta^{*}<\eta,\end{cases} \\
& \mu= \begin{cases}\frac{\mu_{1}}{\mu_{2}} & 0 \leq \eta<\eta^{*} \\
1 & \eta^{*}<\eta,\end{cases}
\end{aligned}
$$

The kinematic equation of the free surface is written in the boundary layer coordinates

$$
\frac{4 \xi}{u} \frac{\partial \eta^{*}}{\partial \tau}+\frac{\partial \eta^{*}}{\partial \xi}=\frac{1}{\xi}\left(\frac{v}{u}-\eta^{*}\right)
$$

\section{Asymptotic solution for large $\xi$}

Here we exhibit a special solution of our coupled air-water system which will be shown later to be a global attractor of non-similar boundary layer solutions. We start by making several assumptions. First we assume that for large $x$ (large $\xi$ ) the horizontal velocity component in the water is linear in $y$, and there is a similarity solution in the air with $f(0)=f^{\prime}(0)=0$, and $f^{\prime \prime}(0)$ is constant. In the air we write

$$
u=f^{\prime}(\eta), \eta=k \frac{y}{x^{1 / 2}}
$$

and in the water

$$
u=c(x) y, Q=\int_{0}^{h} u d y=\frac{c(x)}{2} h^{2}=\text { constant. }
$$

Secondly we assume that the interface position $\eta^{*} \rightarrow 0$ as $\xi \rightarrow \infty$. This assumption is equivalent to assuming that as $\xi \rightarrow \infty$ the liquid layer is a vanishingly small fraction of the boundary layer in the air.

We are going to show that the continuity of the shear stress across the interface $\eta^{*}$ implies that $c=A x^{-1 / 2}$, with $A$ a constant, so that $u=A \eta$ can be expressed in terms of $\eta$ alone. The interface is on $y=h(x), \eta=\eta^{*}=k \frac{h}{x^{1 / 2}}$. The shear stress is continuous

$$
\mu_{1} \frac{\partial u}{\partial y}=\mu_{2} \frac{\partial u}{\partial y} \text { at } \eta^{*}=k \frac{h}{x^{1 / 2}} .
$$

Hence

$$
\mu_{1} c(x)=\mu_{2} f^{\prime \prime}\left(\eta^{*}\right) \frac{\partial \eta}{\partial y}=\mu_{2} \frac{k}{x^{1 / 2}} f^{\prime \prime}\left(\frac{k h}{x^{1 / 2}}\right) .
$$

Now for large $x$, we have assumed that $\eta^{*}=h(x) / x^{1 / 2} \rightarrow 0$, so that $f^{\prime \prime}\left(\eta^{*}\right) \rightarrow f^{\prime \prime}(0)$ which is constant. Then

$$
c(x)=\frac{\mu_{2} k f^{\prime \prime}(0)}{\mu_{1} x^{1 / 2}}=\frac{A}{x^{1 / 2}} .
$$

Using (13), we may write

$$
h^{2}=\frac{2 Q}{A} x^{1 / 2} \stackrel{\text { def }}{=} B^{2} x^{1 / 2}
$$




$$
h=B x^{1 / 4} .
$$

Using (18), we find that

$$
\eta^{*}=\frac{k B x^{1 / 4}}{x^{1 / 2}}=\frac{k B}{x^{1 / 4}} \rightarrow 0 .
$$

Now

$$
f^{\prime}\left(\eta^{*}\right)=u(h(x), x) .
$$

For large $x, \eta^{*} \rightarrow 0$, so that $f^{\prime}\left(\eta^{*}\right) \rightarrow f^{\prime}(0)=0$. Actually we know that $u$ tends to zero because the mass flux is constant but the flow area (18) keeps increasing. We may also show that $v(h(x), x)$ tends to zero for large $x$. We can do this in different ways. Suppose we multiply the kinematic condition (11) by $\xi u$ and let $\xi \rightarrow \infty$.

$$
v=u \xi \frac{\partial \eta^{*}}{\partial \xi}+u \eta^{*} .
$$

From (19) we know that, since $\xi=k x^{1 / 2}$, as $\xi \rightarrow \infty$,

$$
\eta^{*}=\frac{k^{3 / 2} B}{\xi^{1 / 2}} .
$$

Thus as $\xi \rightarrow \infty$,

$$
\xi \frac{\partial \eta^{*}}{\partial \xi}=-\frac{k^{3 / 2} B}{\xi^{1 / 2}} \rightarrow 0 .
$$

Since $u \rightarrow 0$ as $\xi \rightarrow \infty$, so also $v \rightarrow 0$. So as $\xi \rightarrow \infty$, both $u(h(x), x) \rightarrow 0$ and $v(h(x), x) \rightarrow 0$; that is $f^{\prime}\left(\eta^{*}\right) \rightarrow 0$ and $f\left(\eta^{*}\right) \rightarrow 0$. The interface conditions then become the same as the boundary conditions for a Blasius boundary layer in the absence of the water layer but displaced, and so the velocity in the air is given by the Blasius solution for the boundary layer over a flat plate. The solution in the water $f^{\prime}=A \eta$ also satisfies the Blasius equation $f f^{\prime \prime}+\nu_{2} f^{\prime \prime \prime}=0,0 \leq \eta \leq \eta^{*} \rightarrow 0$ and implies that the jump conditions $[f]=\left[f^{\prime}\right]=0$ may be replaced with the Blasius boundary conditions $f(0)=f^{\prime}(0)=0$ applied upon a solid surface.

This shows that our original assumptions are self-consistent. The reader may verify that the solution (13) with $c(x)$ given by (16) is self-similar (satisfies (24) without the the bracketed term). The main good luck that we have is that $\eta^{*} \rightarrow 0$ for large $x$ even though $h(x) \rightarrow \infty$

\section{Non-similar boundary layers}

Since the asymptotic solution for large $\xi$ is similar, but not in the usual sense, we are motivated to see if this solution can be embedded in a large class of non-similar solutions for large $\xi$ (large Reynolds number $U x / 2 \nu_{2}$ ) and to investigate the possibility that the asymptotic solution enjoys a special status as a global attractor for all solutions of this non-similar family.

Self similar boundary layers depend only on $\eta$ and not on $\xi$. The $\xi$ derivatives of $u, v$ and $\eta^{*}$ may be small, of the order of $\xi^{-n}, n \geq 1$, when $\xi$ is large. We retain those terms in each equation which are $\mathrm{O}(1)$, dropping all terms of $\mathrm{O}\left(\xi^{-n}\right)$, For this computation we assumed 
that the first derivative of $u$ with respect to $\xi$ scale with $\xi^{-1}$, the second derivative with $\xi^{-2}$ and $\frac{\partial \eta^{*}}{\partial \xi}=O\left(\xi^{-3 / 2}\right)$. These scalings can be verified $a$ posteriori. We find that

$$
f f^{\prime \prime}+\nu f^{\prime \prime \prime}-\xi\left(f^{\prime} \frac{\partial f^{\prime}}{\partial \xi}-f^{\prime \prime} \frac{\partial f}{\partial \xi}\right)=0
$$

and at $\eta=\eta^{*}:$

$$
\frac{\partial p}{\partial \eta}=0, \quad\left[f^{\prime}\right]=0,[f]=0,\left[\mu f^{\prime \prime}\right]=0,[p]=[\rho] g \eta^{*}, \quad \frac{\partial \eta^{*}}{\partial \xi}=\frac{1}{\xi}\left(\frac{v}{u}-\eta^{*}\right)
$$

Equation (24) can be found in Schlichting (1987). We may remark that the contributions of the viscous terms in the normal stress balance are $\xi^{-2}$ times the terms retained and the surface tension terms are $\xi^{-3}$ times those retained. 


\section{Solutions of initial value problems for the non-similar, two-fluid boundary layer equation}

\subsection{Evolution of a profile which is initially parabolic in segments.}

Since the non-similar solutions depend on $\xi$, we are obliged to consider the evolution of flows prescribed at some initial position $\xi=\xi_{0}$. Fortunately our simulations of those initial value problems indicate a rapid decay from initial values to the asymptotic solution described in $\S 3$. This situation is not unfamiliar. In the classical theory of boundary layers, the Blasius solution, the similarity solution, is a global attractor for all initial value problems which are not similar (see Serrin (1967)). In our case the attractor cannot be self-similar, but the nonsimilar solution of $\S 3$ arises asymptotically, for large $\xi$ from all initial conditions explored. The first initial condition we used to solve (24) and (25) is plotted below.

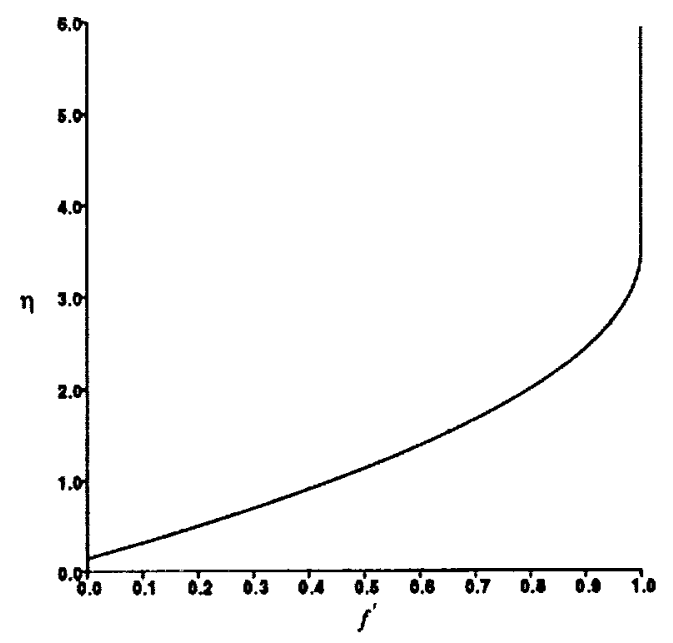

(a)

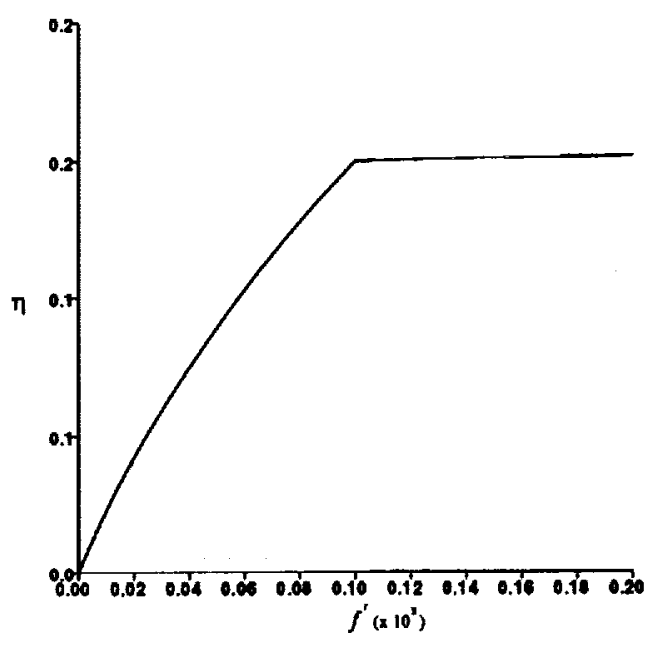

(b)

Figure 1. Parabolic initial condition assumed for the horizontal velocity component $f^{\prime}$ with $\eta^{*}=0.15$ and $\xi_{0}=50$. (a) Initial condition. (b) Detailed plot of initial condition at the interface.

For this initial condition $\eta^{*}=0.15$ and $\xi_{0}=50$. These values were chosen to be representative of conditions discussed by Hastings and Manuel (1985), in which they describe the results of their wind tunnel measurements of a wing in simulated rain. In the above profile, for $0<\eta<\eta^{*}, f^{\prime}$ is parabolic with $f^{\prime \prime \prime}$ being positive. For $\eta^{*}<\eta<3.5, f^{\prime}$ is parabolic with $f^{\prime \prime \prime}$ being negative, and for $\eta>3.5, f^{\prime}=1.0$.

We solved (24) and (25) subject to the aformentioned initial conditions for $\xi>\xi_{o}$ using a finite difference scheme found in Schlichting (1987). This scheme is iterative using secondorder differencing for the $\eta$ derivatives, and a first-order, implicit Euler differencing for the $\xi$ derivatives. We assumed an interface $\eta^{*}=a \xi^{n}$, where $a=\eta^{*}\left(\xi_{o}\right) / \xi_{o}^{n\left(\xi_{o}\right)}$ and found the $n(\xi)$ given in figure 2. 


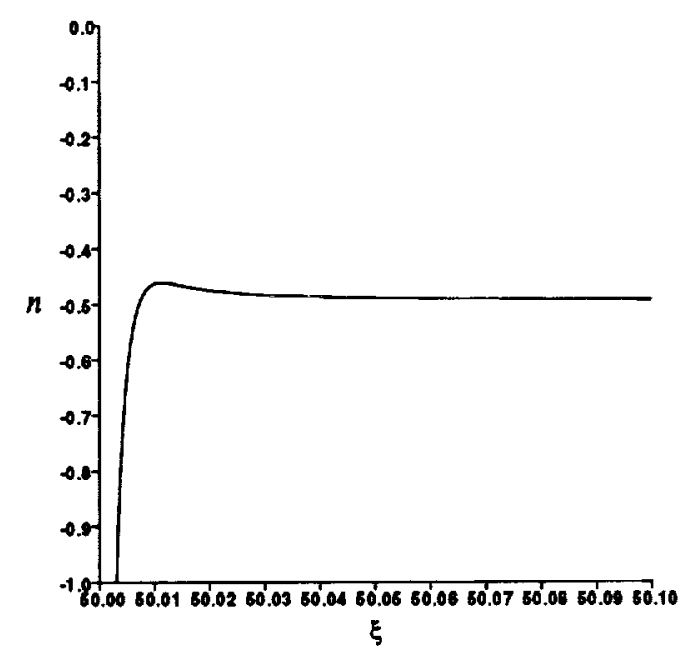

Figure 2. Plot of $n$ for the non-similar boundary layer with initial conditions from figure 1 .

The exponent function $n(\xi)$ decays rapidly to $-1 / 2$, corresponding to a limiting power law increase $h(x) \propto x^{1 / 4}$ of the interface height as given by the asymptotic solution shown in figure 1. For $\xi$ not much larger than $\xi_{0}$ the horizontal velocity component $f^{\prime}$ becomes linear in $\eta, f^{\prime}=c(\xi) \eta$, in the water layer and remains so for all greater $\xi$. The shear stress in the water, $\frac{\mu_{1}}{\mu_{2}} c$, is directly related to the shear stress in the air at the interface through the tangential stress condition found in (25). From our calculations we find that the shear stress in the air at the interface, $f^{\prime \prime}$, asymptotically tends to the shear stress in the Blasius boundary layer of a single fluid over a flat plate at the plate surface, as shown in figure 3 .

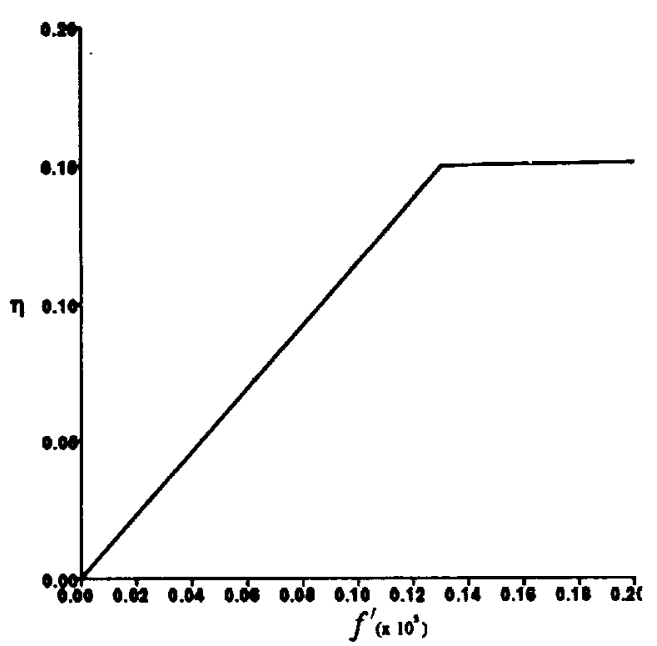

(a)

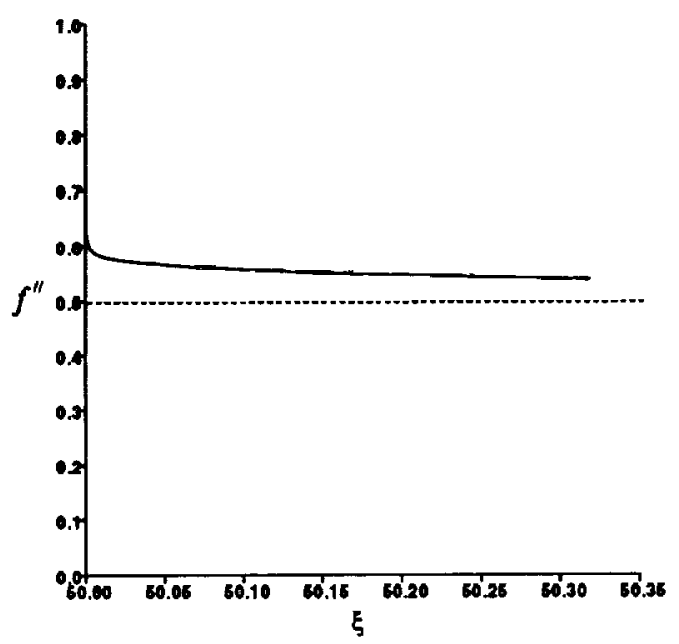

(b)

Figure 3 (a) Detailed plot of boundary layer solution in the water layer at $\xi=50.0675$ for

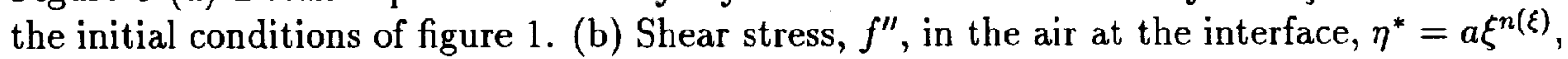
for $\xi>\xi_{o}$.

Notice that the exponent function $n(\xi)$ tends to its asymptotic value extremely rapid, while 
the shear stress at the interface in the air $f^{\prime \prime}$ has a much slower decay. The reason for this difference is $n(\xi)$ becomes nearly $-1 / 2$ as soon as the horizontal velocity component in the water becomes linear, which occurs for $\xi$ not very much greater than $\xi_{0}$. Figure 3 shows that the flow has not yet reached its asymptotic form, since the shear stress at the interface in the air is appreciably different from its asymptotic value. The flow then slowly tends to its asymptotic state. The flow is non-similar for finite $\xi$ because the boundary layer height and the interface do not scale the same. Asymptotically one may say the flow is similar because $\eta^{*}$ goes to zero and the flow in the air is the self-similar Blasius profile.

\subsection{Evolution of another profile which is initially parabolic in segments.}

In the next example we used the same initial $f^{\prime}(\eta)$ for $\eta>\eta^{*}$ as in figure 1(a), but the profile shown in figure 1(b) was replaced with the one shown in figure 4.

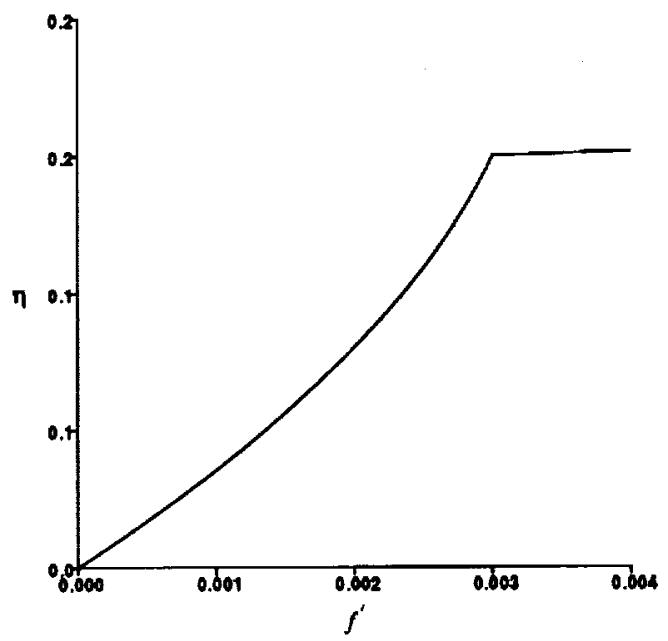

Figure 4. Initial profile in the water at $\xi=\xi_{0}$. The profile in the air is shown in figure 1(a).

In figure 5 we show the evolution of the exponent $n(\xi)$ for $\xi>\xi_{0}$ for the aformentioned initial condition. Obviously $n(\xi) \rightarrow-1 / 2$ for large $\xi$ and $\eta^{*}=a \xi^{-1 / 2}$ for large $\xi$. The solution given in $\S 3$ is attained asymptotically. 


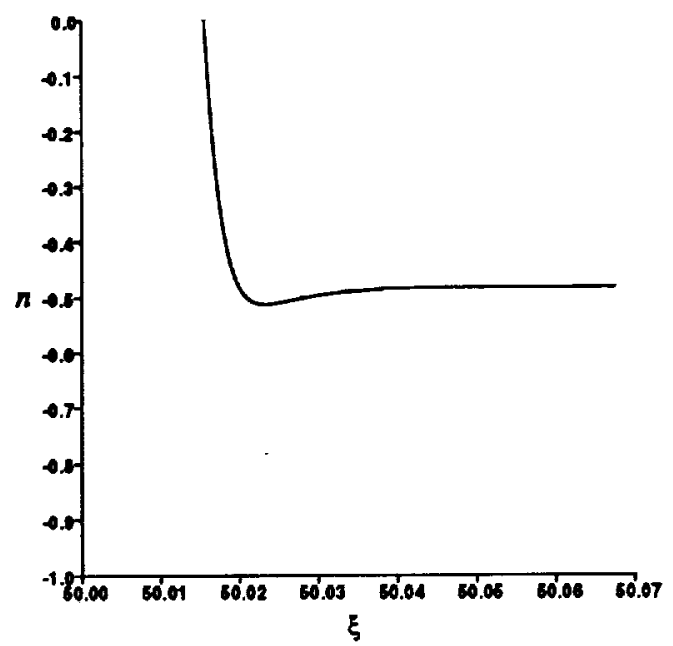

Figure 5. Exponent function $n(\xi)$ for $\eta^{*}=a \xi^{n(\xi)}$.

\subsection{Evolution of a profile which is initially in similarity form but with $\eta^{*}\left(\xi_{o}\right)=0.15>0$.}

The initial condition shown in figure 6 is generated from (24) and (25) when the $\xi$ derivatives are set to zero, so that

$$
f f^{\prime \prime}+\nu f^{\prime \prime \prime}=0
$$

and at $\eta=\eta^{*}:$

$$
\frac{\partial p}{\partial \eta}=0, \quad\left[f^{\prime}\right]=0,[f]=0, \quad\left[\mu f^{\prime \prime}\right]=0, \quad[p]=[\rho] g \eta^{*} .
$$

This system is solved using a iterative finite difference scheme using second order differencing and gives rise to the profiles shown in figure 6 .

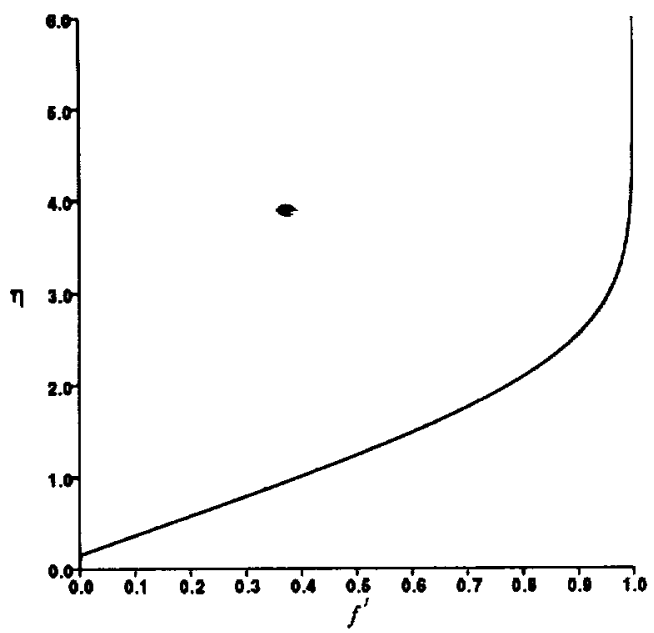

(a)

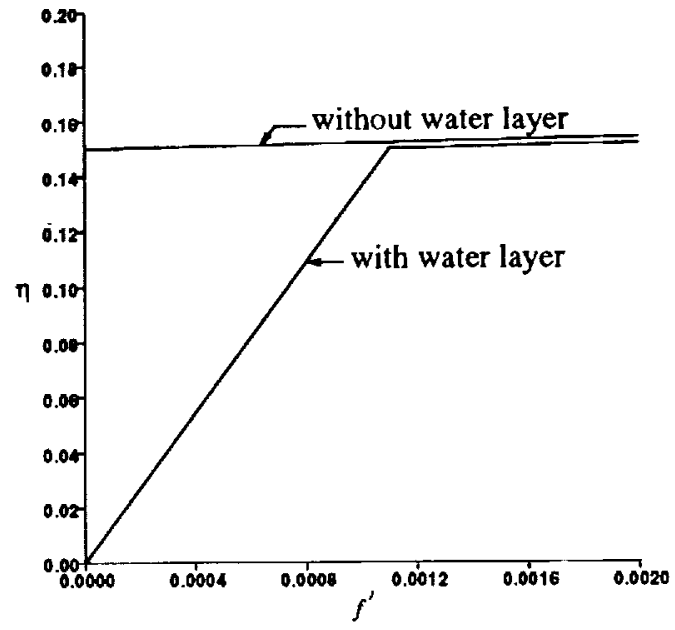

(b)

Figure 6. Solution $f^{\prime}(\eta)$ of (26) and (27). (a) $\eta>0.15$. (b) $\eta<0.15$. The Blasius solution of (24) for $f(0.15)=f^{\prime}(0.15)=0$ is also shown in (b). 
The profiles in figure 6 were used for initial values in the non-similar equations (24) and (25) and they evolved to the coupled self-similar solutions described in $\S 3$ in which $\eta^{*}(\xi) \rightarrow 0$. The difference between the solution in the air and the classical Blasius flat plate solution also tends to zero. A graph of the exponent function for this example is shown figure 7 .

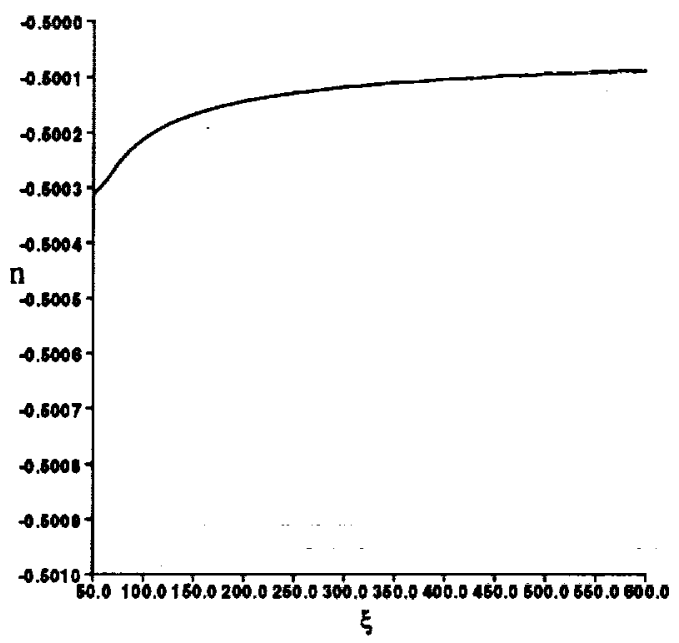

Figure 7. Exponent function $n(\xi)$ for $\eta^{*}=a \xi^{n(\xi)}$ for the initial conditions of figure 6 .

In these examples and in all the others, which we tried but are not shown, the asymptotic solution given in $\S 3$ is ultimately attained.

\section{Behavior of $\xi$ derivatives of the non-similar solution}

In deriving the system which lead to the above solutions, we assumed that first and second derivatives of $u$ and $\eta^{*}$ with respect to $\xi$ were inversely proportional to $\xi^{-n}, n \geq 1$. ¿From our solution we find that $\frac{\partial \eta^{*}}{\partial \xi}$ scales like $\xi^{-3^{\prime 2}}$ and $\frac{\partial^{2} \eta^{*}}{\partial \xi^{2}}$ scales like $\xi^{-5 / 2}$, in agreement with our scalings. Since $\xi$ scales with $x^{1 / 2}$, the interface position scales with $x^{1 / 4}$.

In order to examine the $\xi$ derivatives of $u$, we define two functions,

$$
\begin{gathered}
F_{1}(\eta, \xi)=\xi \frac{\partial f^{\prime}}{\partial \xi} \\
F_{2}(\eta, \xi)=\xi^{2} \frac{\partial^{2} f^{\prime}}{\partial \xi^{2}} .
\end{gathered}
$$

Depending upon the initial conditions chosen at $\xi_{o}, F_{1}\left(\eta, \xi_{o}\right)$ and $F_{2}\left(\eta, \xi_{o}\right)$ may be extremely large. However, for larger values of $\xi$ the horizontal velocity component in the water becomes linear in $\eta, n(\xi)$ tends to $-1 / 2$ and $F_{1}(\eta, \xi)<1, F_{2}(\eta, \xi)<1$ (see figure 8). 

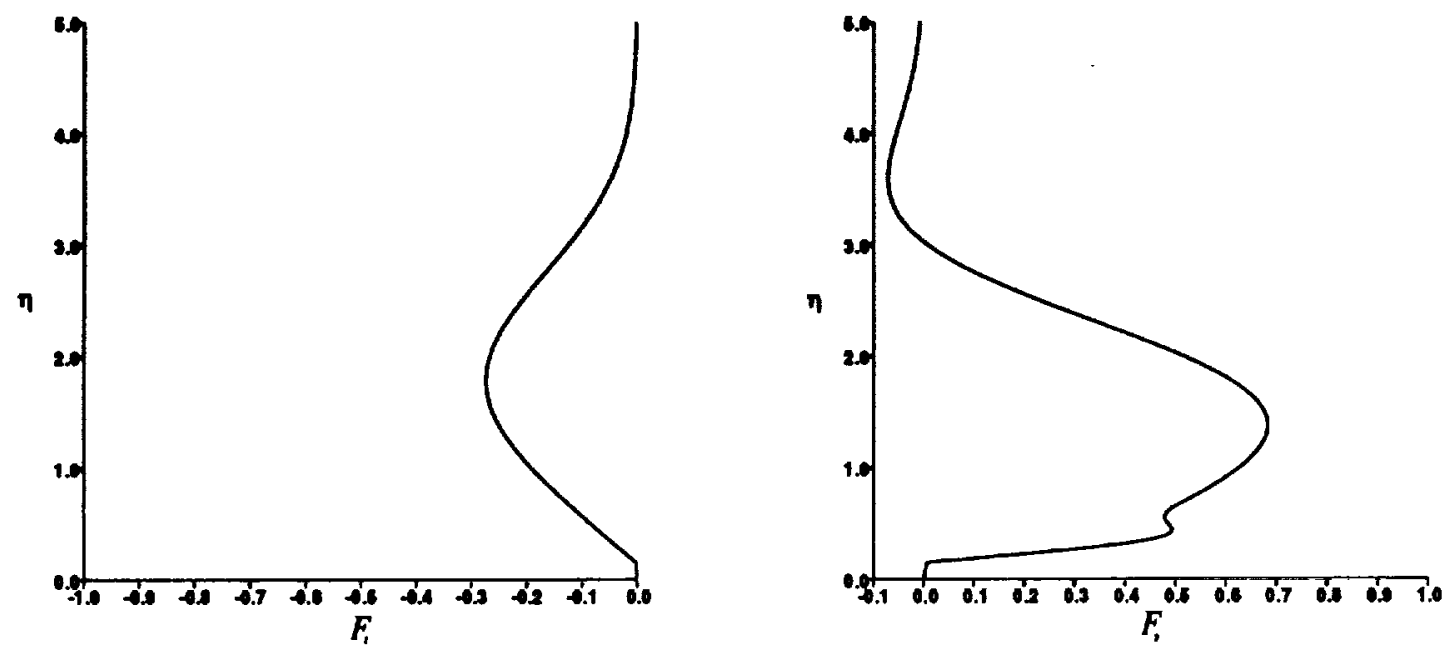

Figure 8. Plot of $F_{1}(\eta, \xi)$ and $F_{2}(\eta \xi)$ versus $\eta$ for $\xi=50.01$ for the flow plotted in figure 6 .

$\left|F_{1}\right|$ and $\left|F_{2}\right|$ decrease, as shown in figure 9, although the decrease in $\left|F_{1}\right|$ is very small. Since as $\xi \rightarrow \infty, \eta^{*} \rightarrow 0$ and the flow in the air goes to the Blasius boundary, both $F_{1} \rightarrow 0$ and $F_{2} \rightarrow 0$ as $\xi \rightarrow \infty$.
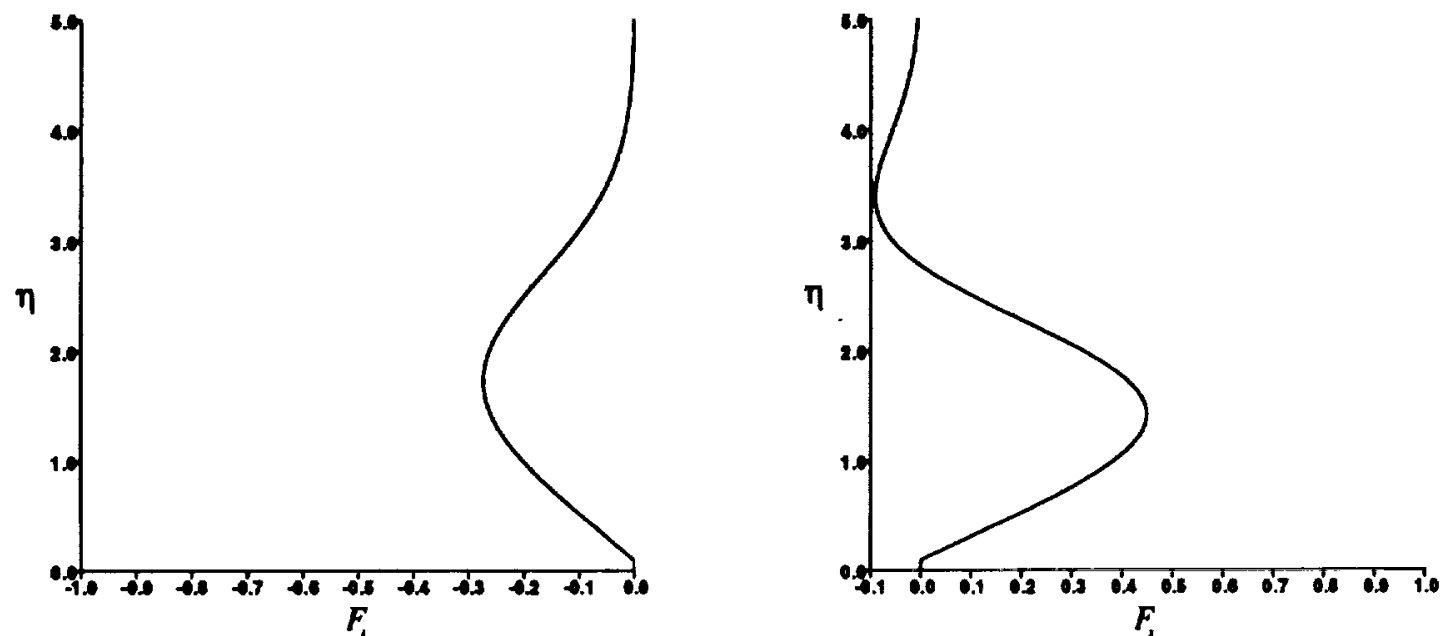

Figure 9. Plot of $F_{1}(\eta, \xi)$ and $F_{2}(\eta \xi)$ versus $\eta$ for $\xi=129.1$ for the flow plotted in figure 6 .

Then since $F_{1}$ and $F_{2}$ are both $\mathrm{O}(1)$, the assumptions that were made in deriving (24) and (25) are shown to hold. 


\section{Concluding remarks}

The analysis given in this paper can be extended to two-fluid boundary layer problems with other free streams, say $U=U_{\infty} x^{n}$. It is also probable that the solution given in $\S 3$ and the other limiting solutions to which we have just alluded are unique large $x$ limits of steady coupled air-water solutions of the Navier-Stokes equations with different initial profiles at $x=x_{o}$.

\section{REFERENCES}

E. C. Hastings Jr. and G. S. Manuel, "Measurements of water film characteristics on airfoil surfaces from wind tunnel tests with simulated heavy rain," AIAA Paper 85-0259 (1985). J. Serrin, "Asymptotic behaviour of velocity profiles in the Prandtl boundary layer theory", Proc. R. Soc. Lond. A 299, $491-507$ (1967).

H. Schlichting, Boundary-Layer Theory (McGraw-Hill, New York, 1987), 7th ed., pp. 187191.

C.Y. Wang "The boundary layers due to shear flow over a still fluid," Phys. Fluids A 4, 1304 (1992)

C.-S. Yih "Wave formation on a liquid layer for de-icing airplane wings," J. Fluid Mech. 212, 41 (1990) 


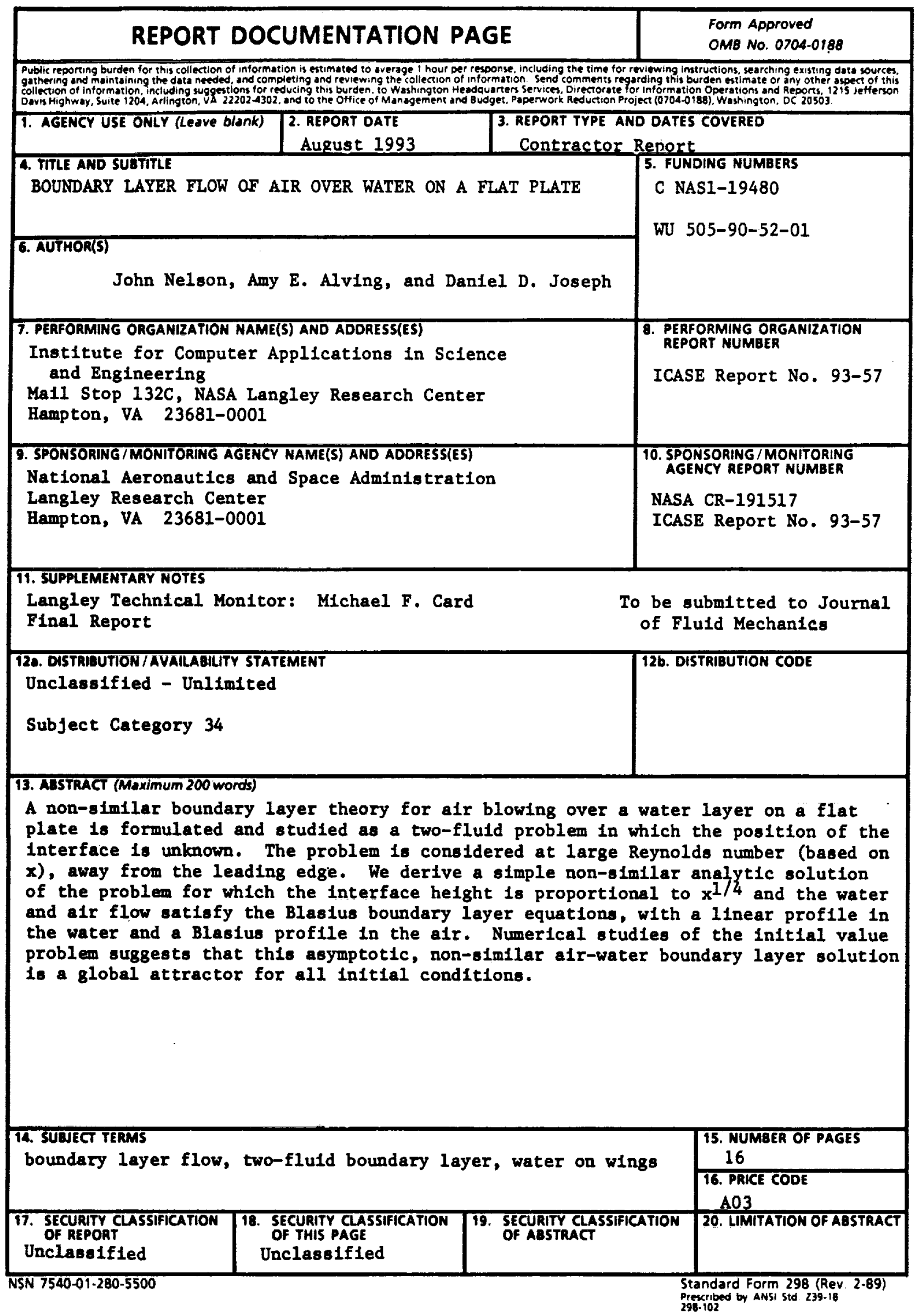


National Aeronautics and

Space Administration

Code JTT

Washington, D.C.

20546-0001

Official Business

BULK RATE

POSTAGE \& FEES PAID NASA

Permit No. G.27

Penalty for Private Use, $\mathbf{\$ 3 0 0}$ 